\title{
Predicting Toxicity of Food-Related Compounds Using Fuzzy Decision Trees
}

\author{
Daishi Yajima, Takenao Ohkawa, Kouhei Muroi, and Hiromasa Imaishi
}

\begin{abstract}
Clarifying the interaction between cytochrome P450 (P450) and food-related compounds that affect the metabolic activity of $\mathbf{P 4 5 0}$ allows to effectively predict the toxicity of food-related compounds. Currently, we are developing a fluorescence $\mathbf{P 4 5 0}$ sensor to evaluate the metabolic reactions of food-related compounds. The amount of fluorescent metabolic products for various $\mathbf{P 4 5 0}$ species can be easily measured using sensors.

In this paper, a method of constructing models to predict toxicity of food-related compounds from the amount of fluorescent metabolic product using a machine learning technique is proposed. Since the precision of the measurement of the amount of fluorescent metabolic products is not high enough to quantitatively predict the toxicity value, multiple regression analysis is not always appropriate. We consider the toxicity prediction problem to be a toxicity classification problem. In this framework, however, it is difficult to determine the precise boundary values for separating one toxicity class from another. In our proposed method, fuzzy sets are introduced not only to the attributes for dividing instances but also to the classes that give the prediction results to solve the difficulty of determining the boundary values. The performance of our proposed method was confirmed by comparing the mean error and the cosine similarity with those of other methods.
\end{abstract}

Index Terms-Fluorescence sensor, fuzzy decision tree, $\mathbf{P 4 5 0}$, toxicity prediction.

\section{INTRODUCTION}

In recent years, as the development of such food-related compounds as functional food and food additives has been promoted, the safety assessment of food-related compounds has become more important. Instrumental analysis and animal experiments are usually used for such assessments. However, applying the results obtained by animal experiments to humans is not always appropriate, because the metabolic activation capability depends on animal species or physiological differences between males and females. In addition, it is difficult to address the interaction among the great varieties of food compounds by such methods, which also require high cost and a long time to get results. In recent years, a quite new technology for safety assessment has been developed by focusing on the $\mathrm{P} 450$ enzymes that perform the metabolic activation of food-related compounds. The metabolic activation is a phenomenon in which nontoxic

Manuscript received July 5, 2013; revised September 25, 2013

Daishi Yajima and Takenao Ohkawa are with the Graduate School of System Informatics, Kobe University, Kobe, Japan (e-mail: yajima@cs25.scitec.kobe-u.ac.jp, ohkawa@kobe-u.ac.jp ).

Kouhei Muroi is with the Graduate School of Agricultural Science, Kobe University, Kobe, Japan (e-mail: a0873_3826@yahoo.co.jp).

Hiromasa Imaishi is with the Research Center for Environmental Genomics, Kobe University, Kobe, Japan (e-mail: himaish@kobe-u.ac.jp). compounds become toxic metabolites by metabolism in human body. Specifying the interaction between P450 and food compounds that affect the reaction of metabolic activity allows us to effectively predict the toxicity of food compounds. An oxygen sensor measurement system has been developed that can directly evaluate the metabolic reaction between food compounds and P450, and predicting mutagenicity has been attempted by only analyzing the reaction patterns of enzymes obtained by sensors [1] However, oxygen sensors show extremely low sensitivity and require a precise measurement environment in which the inflow of oxygen has to be blocked completely. As an alternative to oxygen sensors, we are developing a fluorescence sensor to evaluate the metabolic reactions of foods more sensitively and easily. Therefore, a model is required that predicts the toxicity from the amount of fluorescent metabolic product for each $\mathrm{P} 450$.

In this paper, a method of constructing models that can predict the toxicity of food-related compounds from fluorescence sensor data using a machine learning technique is proposed. Multiple regression analysis is often used to estimate a formula that predicts the value of an objective variable (toxicity) from continuous variables (amount of fluorescent metabolic product). However, since the precision of the measurement of the amount of fluorescent metabolic products is not high enough to quantitatively predict the toxicity value, multiple regression analysis is not always appropriate. Therefore, we consider the toxicity prediction problem to be a toxicity classification problem, in which each food compound is classified into a toxicity class, such as high or low. In the classification problem, each toxicity class has to be defined in advance. However, it is difficult to decide the precise boundary values for separating one toxicity class from another. To cope with this problem, we introduce a fuzzy decision tree.

Many approaches have constructed fuzzy decision trees. Lee et al. constructed a fuzzy decision tree for fuzzy data [2]. This method can automatically generate membership functions for continuous numeric attributes. Yuan and Shaw constructed a fuzzy decision tree that focused on ambiguity [3]. This method evaluated how suitably the value belongs to each attribute and then the attribute with minimum ambiguity is selected to divide instances.

Fuzzy decision trees ambiguously classify instances by introducing fuzziness to the attributes. In our proposed method, fuzzy sets are introduced not only to the attributes for dividing instances but also to the classes that give the prediction results, since it is difficult to strictly decide the boundaries to discriminate between toxicity classes.

The remaining sections are organized as follows. Section II describes the data obtained from umu tests and a 
fluorescence sensor. Section III presents a fuzzy decision tree algorithm for toxicity prediction. The evaluation of our experimental result is shown in Section IV. Two types of experiments are performed in terms of different points. Finally Section V provides discussions and conclusions of this study.

\section{UMU TEST AND SIGNATURES}

\section{A. Genotoxicity Assessment by Uти Test}

P450 enzymes are metabolic activators for food-related compounds, such as food additives and mycotoxins. By metabolic activation, compounds with no toxicity often become toxic metabolites. Fifty seven types of P450 in humans have been identified [4]. The umu test quantitatively evaluates the reaction of metabolic activation using P450 in rats. The results of umu tests are conventionally classified into three categories. If the value is less than 1.0, the compound does not greatly affect our bodies. If it is more than 2.0, the compound shows toxicity in the body, that is the compound has a mutagenic property. If it is between 1.0 and 2.0, the compound has some influence on our bodies. Boundary values 1.0 and 2.0 are usually used for convenience. However, they have been defined not from theoretical evidence but from experiential aspects.

Examples of toxicity values are shown in Table I.

\begin{tabular}{ll}
\multicolumn{2}{c}{ TABLE I: UMU TEST RESULTS } \\
\hline \hline Name of compound & Value of umu test \\
\hline Caffeine & 1.28 \\
Feric acid & 0.98 \\
Methylegenol & 1.65 \\
Linalool & 1.26 \\
Cineol & 1.22 \\
\hline \hline
\end{tabular}

\section{B. Signature from Fluorescence Sensors}

Umu tests have problems: high cost and long processing. On the other hand, sensors have been developed that can measure the amount of enzymatic metabolites by $\mathrm{P} 450$ enzymes, and the data obtained from them can be applied to evaluate the toxicity of food-related compounds [5]-[7].

\begin{tabular}{cccccc}
\multicolumn{6}{c}{ TABLE II: SIGNATURE } \\
\hline \hline \multicolumn{5}{c}{ Kind of P450 } \\
\hline $\begin{array}{c}\text { Name of } \\
\text { compound }\end{array}$ & $\begin{array}{c}\text { Presence or absence of } \\
\text { compound }\end{array}$ & $2 \mathrm{C} 8$ & $3 \mathrm{~A} 4$ & $\cdots$ & $\begin{array}{c}\text { C } 1 \\
8\end{array}$ \\
\hline \multirow{4}{*}{ Caffeine } & Without compound & 579 & 647 & $\cdots$ & 2234 \\
& & 1 & 2 & & \\
& With compound & 511 & 743 & $\cdots$ & 2149 \\
\hline \hline
\end{tabular}

Several P450 enzymes are located on one fluorescence sensor plate, and the intension of the fluorescence of fluorescent metabolites that is metabolized by each P450 can be simultaneously measured with the fluorescence sensor. Competitive enzymatic reaction between a food-related compound and each $\mathrm{P} 450$ are measured using the fluorescence sensor, and the results are viewed as a competitive enzymatic reaction that consists of all the enzyme reactions, called a signature for this compound. An example of a signature for a food-related compound, caffeine, is shown in Table II. "Without compound" indicates the amount of the product generated by metabolizing the fluorogenic substrate by each P450, and "with compound" means the amount of fluorescent metabolites when caffeine inhibits the metabolism of the fluorogenic substrate. The safety of the compounds is evaluated at low cost and quickly by predicting the toxicity from the signature.

\section{PROPOSED METHOD}

\section{A. Construction of a Fuzzy Decision Tree}

As mentioned above, we consider a toxicity assessment problem to be a toxicity classification problem. In the classification problem, several classes, which each instance is classified into, have to be defined beforehand based on the intensity of the toxicity. To define the classes, the boundary values of the toxicity are required to distinguish each class, but they cannot be decided theoretically.

In general, constructed decision trees provide good evaluations, if the correct classes for the given instances are predicted accurately. Since the values of the boundaries between classes are not absolute, however, it is not appropriate that only one class is deemed a correct class for the instances that are positioned near the boundaries. So the concept of fuzzy decision trees was introduced.

A fuzzy decision tree can handle data ambiguity. In a fuzzy decision tree, the attributes that describe each instance are defined using fuzzy sets. A fuzzy set is defined as a set whose elements have degrees of membership [8]. Fuzzy decision trees can show the degree to which an instance belongs to a class, instead of showing an instance that belongs or does not belong to a class. In them, fuzzy sets are usually used as attributes to represent instances. Additionally, in our method, they are used as a class to which an instance belongs.

An algorithm for constructing a fuzzy decision tree is shown in Fig. 1:

1. Classify each instance $e_{j}$ of training set $E$ into multiple fuzzy classes with confidence degree $c d$, for $j=1, \ldots,|E|$.

2. Assign $E$ to $N_{\text {root }}$, where $N_{\text {root }}$ is a root node.

3. In current node $N$, If degree in separation exceeds threshold value $t 1$ or sum of $c d$ falls is below $t 2$, $N$ is labeled percentage of each class and is regarded as a leaf.

4. otherwise
a. Select attribute $a_{j}$ and boundary value $t$ to divide.
b. Make fuzzy sets $F_{A_{1}}, F_{A_{2}}$.
c. Divide $E_{N}$ to child nodes $N_{\text {child }}, N_{\text {child }}$ according to $F_{A_{1}}, F_{A_{2}}$, where $E_{N}$ is a set of instances in $N$.
d. For each child node go to step 3 .

Fig. 1. Algorithm.

In this algorithm, the fuzzy classes to which an instance in the training set belongs are decided using membership functions; they provide the grade value in the interval $[0,1]$ for each class based on the toxicity values in step 1 . In this study, trapezoidal membership function $\mu_{C}(x, \alpha, \beta, \gamma, \delta)$ is used to simplify the calculation [2]: 


$$
\mu_{C}(x, \alpha, \beta, \gamma, \delta)=\left(\begin{array}{ll}
0 & x \leq \alpha \\
\frac{x-\alpha}{\beta-\alpha} & \alpha<x<\beta \\
1 & \beta \leq x \leq \gamma \\
\frac{x-\delta}{\gamma-\delta} & \gamma<x<\delta \\
0 & \delta \leq x
\end{array}\right.
$$

An instance, which can belong to more than one fuzzy class, is given a grade value as confidence degrees (degree of belonging to the class) for each fuzzy class. Thus an instance belonging to two fuzzy classes is regarded as two instances that belong to each class. After step 1 is applied to all the instances in the training set, all are assigned to the root node in step 2. Parameter $t 1$ introduced in step 3 is a threshold for the degree of separation, defined as follows:

$$
v(S)=\max _{i} \frac{\left|S_{c_{i}}\right|}{|S|},
$$

where $c_{i}$ is an $i$-th class of class set $C$ and $|S|$ is the sum of the confidence degrees of the instances included in instance set $S$. The set of instances is usually divided into more than one subset to get a higher degree of separation. However, if the current degree of separation exceeds $t 1$, the instances are not divided. Parameter $t 2$ is the threshold for the sum of the confidence degrees, and if the sum of the confidence of the current node is less than $t 2$, the set of instances is not divided any more.

In step $4 \mathrm{a}$, the dividing attribute and the boundary value are selected. To determine the attribute for the dividing instances, our proposed method uses a gain ratio, which is calculated as follows based on a conventional manner [9].

First, the gain is defined as

$$
\operatorname{gain}(A, X)=E(S)-E(S \mid A),
$$

where $E(S)$ is the entropy for all the instances in a node and $E(S \mid A)$ is the entropy for the case where the set of instances is divided into subsets by attribute $A$. Entropy $E(S)$ of a set of training instances in a node is defined as

$$
E(S)=-\sum_{i=1}^{t} X\left(c_{i}\right) \log _{2} X\left(c_{i}\right)
$$

where $X\left(c_{i}\right)$ is the ratio of the sum of the confidence degrees of instances that belong to $c_{i}$ to the sum of the confidence degree of all the instances in the node. Entropy $E(S \mid A)$ is defined as

$$
E(S \mid A)=-\sum_{j=1}^{u} X\left(a_{j}\right) \sum_{i=1}^{t} X\left(c_{i} \mid a_{j}\right) \log _{2} X\left(c_{i} \mid a_{j}\right),
$$

where $a_{j}$ is the $j$-th attribute value of attribute $A$ and $X\left(c_{i} \mid a_{j}\right)$ is a ratio of the sum of the confidence degrees of instances belonging to $c_{i}$ and having $a_{j}$ to that of the instances having $a_{j}$.
Finally, when the set of instances is divided into subsets by attribute $A$, information gain ratio gain_ratio $(A, S)$ is defined as

$$
\operatorname{gain}_{-} \operatorname{ratio}(A, S)=\frac{\operatorname{gain}(A, S)}{\operatorname{divide}(A, S)},
$$

where $\operatorname{divide}(A, S)$ is the amount of divided information obtained by dividing the set of instances, defined as

$$
\operatorname{divide}(A, S)=-\sum_{u=1}^{t} X\left(a_{j}\right) \log _{2} X\left(a_{j}\right) .
$$

The numeric attribute domains are discretized by determining the boundary points. Numerical attribute value $a$ is divided into attribute values $\{t<a\}$ and $\{a \leq t\}$ by boundary value $t$. The gain ratio is calculated for all the attributes, and an attribute (a dividing attribute) and a boundary value are selected to maximize the gain ratio.

It is insufficient to consider the ambiguity of the continuous attributes that are divided into two crisp values $\{a$ $\leq t\},\{t<a\}$ at boundary value $t$ of selected attribute $A$. Therefore, fuzzy sets are generated to ambiguously classify instances with attribute values near the boundary values as follows:

$$
\begin{aligned}
& \mu_{A_{1}}(x, t-w, t+w)=\left(\begin{array}{ll}
1 & x \leq t-w \\
\frac{-x+t+w}{2 w} & t-w<x<t+w \\
0 & t+w \leq x
\end{array}\right. \\
& \mu_{\mathrm{A}_{2}}(x, t-w, t+w)=\left(\begin{array}{ll}
0 & x \leq t-w \\
\frac{x-t+w}{2 w} & t-w<x<t+w \\
1 & t+w \leq x
\end{array}\right.
\end{aligned}
$$

As stated above, boundary value $t$ is decided that maximizes the gain ratio. Then two fuzzy sets with membership functions $\quad \mu_{A_{1}}(x, t-w, t+w) \quad$ and $\mu_{A_{2}}(x, t-w, t+w)$ are generated using (8) and (9) to ambiguously evaluate an instance that has an attribute value in the range $\pm w$ from boundary value $t$, where $w$ is a parameter given in advance. If an instance is divided based on the generated fuzzy sets, a grade provided by each set is calculated by the membership functions, and the confidence degree is updated:

$$
n e w_{-} c d=n o w_{-} c d \times \text { grade },
$$

where now_cd represents the confidence degree before the update and new_cd represents it after the update. If the confidence degree of an instance shows 0.0 , it is removed from the instance set. Steps 3 and 4 are recursively applied to the set of instances in the child node.

\section{B. Toxicity Prediction by the Fuzzy Decision Trees}

The signature obtained from the fluorescence sensors is used as input data. The difference between the amount of 
fluorescent products metabolized with and without a compound is used as the value corresponding to each $\mathrm{P} 450$ enzyme in the input signature. An example of input data is shown in Table III.

\begin{tabular}{cccccc}
\multicolumn{5}{c}{ TABLE III: INPUT DATA } \\
\hline \hline \multicolumn{5}{c}{ Kind of P450 } \\
\hline $\begin{array}{c}\text { Name of } \\
\text { compound }\end{array}$ & $2 \mathrm{C} 8$ & $3 \mathrm{~A} 4$ & $\cdots$ & $2 \mathrm{C} 18$ \\
\hline Caffeine & -676 & 958 & $\cdots$ & 266 \\
\hline \hline
\end{tabular}

The degree of belonging to each fuzzy set (class), which is defined based on the intensity of the toxicity, is calculated for the input instance and is presented as a predicted result. For example, if five classes are prepared, the prediction result is shown as $[0.0,0.3,0.5,0.2,0.0]$ and corresponds to [classes 1 , 2, 3, 4, and 5].

Fig. 2 shows an example of the prediction process using fuzzy decision trees. Instance A is given 1.0 as a confidence degree in the beginning and goes down based on first dividing attribute $2 C 8$. For example, if the value of $2 C 8$ of an instance is 20 , the value of attribute $2 C 8$ of the instance is considered "high" with 0.2 of confidence degree by membership functions. The instance whose value of $2 C 8$ is considered "high" goes down to a next node that is labeled $3 A 4$ as a dividing attribute. If the value of $3 A 4$ of the instance is 200 , the value is considered completely "high," and the instance reaches the leaf node labeled class 3 (1.0) with a 0.2 confidence degree. On the other hand, an instance whose value of $2 C 8$ is considered "low" goes down to a next node that is labeled $2 \mathrm{C} 18$ as a dividing attribute, and then the instance goes down based on a branch labeled "low," since the value of $2 C 18$ is -500 , and the instance reaches the leaf node labeled classes $1(0.4), 2(0.4)$, and $3(0.2)$ with a 0.8 confidence degree. Instance $\mathrm{A}$ obtains the following prediction results: $[0.32(=0.8 \times 0.4), 0.32(=0.8 \times 0.4)$, $0.36(=0.2 \times 1.0+0.8 \times 0.2), 0.0,0.0]$.

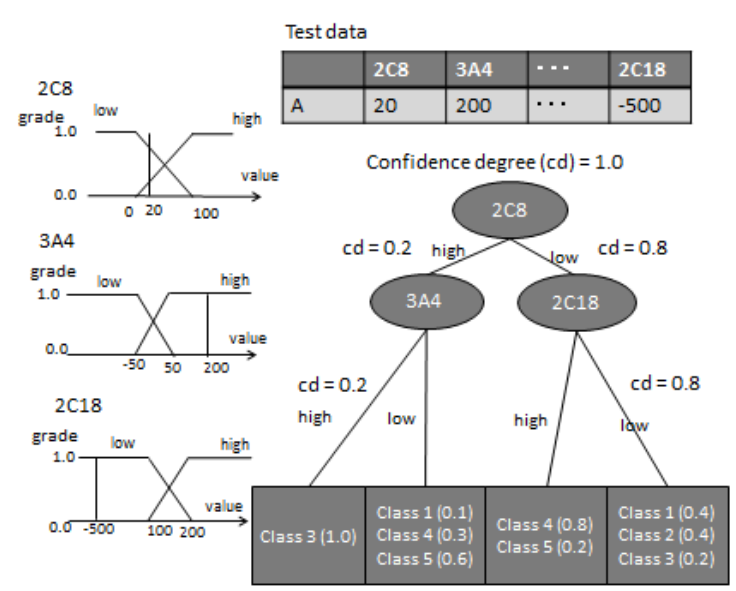

Fig. 2. Example of prediction process in fuzzy decision tree.

\section{EXPERIMENTS}

\section{A. Evaluation Experiments}

In the experiments, twelve important P450 species taking part in drug metabolism in human were used to obtain signatures from a fluorescence sensor. Signatures were obtained four times for each compound. The differences between the amount of fluorescent products metabolized with and without a compound for each P450 enzyme were used as attributes. In addition, the toxicity value examined by the umu test in advance was added. Totally 324 instances were used, since they were obtained four times for each of 94 kinds of compounds, but some instances were removed that were missing values. Examples of instance are shown in Table IV.

\begin{tabular}{c|cccc|c}
\multicolumn{7}{c|}{ TABLE IV: EXAMPLE OF INSTANCES } \\
\hline \hline & \multicolumn{5}{c}{ Kind of P450 } \\
\hline $\begin{array}{c}\text { Name of } \\
\text { compound }\end{array}$ & $2 \mathrm{C} 8$ & $3 \mathrm{~A} 4$ & $\cdots$ & $2 \mathrm{C} 18$ & $\begin{array}{c}\text { Umu } \\
\text { test }\end{array}$ \\
\hline Caffeine & -676 & 958 & $\cdots$ & 266 & 1.28 \\
\hline Catechol & 125 & 22 & & -1222 & 1.47 \\
\hline$\vdots$ & $\vdots$ & $\vdots$ & $\vdots$ & $\vdots$ & $\vdots$ \\
\hline \hline
\end{tabular}

In this paper, our proposed method is evaluated from the following two points.

1) Accuracy of toxicity value prediction

2) Accuracy of class prediction

In the experiments, the number of classes was four, and the other parameters are shown as follows:

- Membership functions for defining the classes:

$$
\begin{aligned}
& \mu_{c_{1}}(x, 0.4,0.7,0.7,1.0) \mu_{c_{2}}(x, 0.6,0.9,0.9,1.5) \\
& \mu_{c_{3}}(x, 0.9,1.3,1.3,1.9) \mu_{c_{4}}(x, 1.5,1.9,1.9,2.1)
\end{aligned}
$$

- Margin from the boundary value on the membership function generated for each attribute: $w=215$

- Threshold of degree of separation: $t 1=0.8$

- Threshold of confidence concentration: $t 2=9.0$

Our proposed method was compared with three conventional methods: multiple regression analysis, a decision tree, and an overlapped decision tree. In the decision tree and in the overlapped decision tree, the classes were defined as follows. The number is just four, like in the proposed method. In the decision tree, the boundary points for distinguishing the classes are the median between two adjacent representative values, which are the gravity points of the membership function for defining classes in our proposed method. The overlapped decision tree allows classes to overlap. The range of each class is $[\alpha, \delta]$ and corresponds to membership function $\mu_{c}(x, \alpha, \beta, \gamma, \delta)$ in our proposed method. The representative points are gravity points.

In our proposed method, each instance belongs to one or more classes with the confidence degree. Similarly, in the decision tree and in the overlapped decision tree, each instance belongs to one class (in the decision tree) or one or more classes (in the overlapped decision tree) with a 1.0 confidence degree.

In the first experiment (experiment 1), the accuracy of predicting the toxicity value was compared for each method using the average error of the predicted value to the actual value obtained from the umu test. For that purpose, the predicted classes of an instance are defuzzified; the classes were quantified by totaling for all the classes what was 
obtained by multiplying the rate at which the instance belongs to a class by its representative value.

In the second experiment (experiment 2), the accuracy of the class prediction was evaluated by cosine similarity, which represents the closeness of the angle between vectors, and is defined as follows:

$$
\cos (\vec{x}, \vec{y})=\frac{\vec{x} \cdot \vec{y}}{|\vec{x}||\vec{y}|}=\frac{\vec{x}}{|\vec{x}|} \cdot \frac{\vec{y}}{|\vec{y}|}=\frac{\sum_{i=1}^{n} x_{i} y_{i}}{\sqrt{\sum_{i=1}^{n} x_{i}^{2}} \cdot \sqrt{\sum_{i=1}^{n} y_{i}^{2}}}
$$

where $n$ represents the number of the dimensions of the vector. The prediction result and the actual classes are represented as a four-dimensional vector that consists of the degree of belonging to each class to apply (11).

In each experiment, a 10 -fold cross validation $(\mathrm{CV})$ was used for comparing the results of the methods. Moreover, the average of ten 10 -fold CVs was also evaluated.

\section{B. Discussion}

Fig. 3 and Fig. 4 show the results of experiment 1. Fig. 3 shows the average error in one trial of a 10 -fold $\mathrm{CV}$ by each method. Fig. 4 shows the result of performing a 10-fold CV ten times, and Table V shows their average.

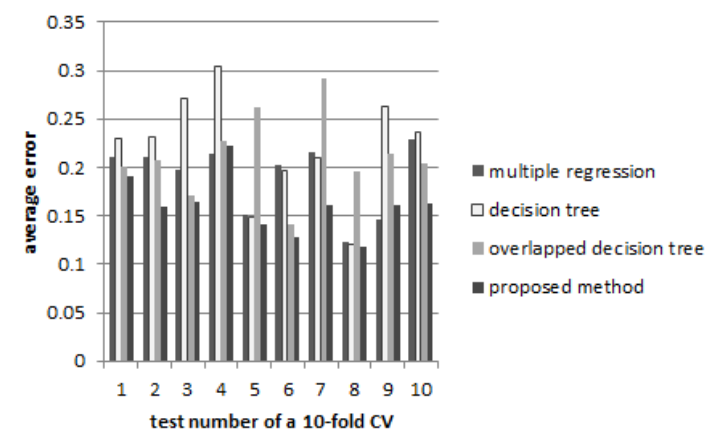

Fig. 3. 10-fold CV results (error).

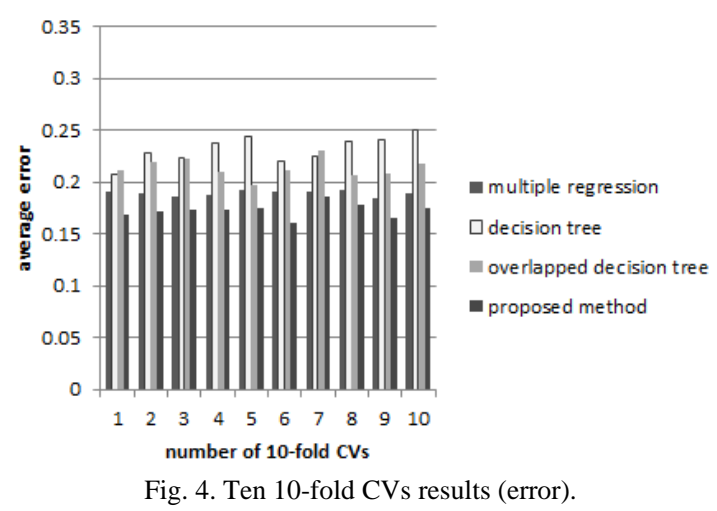

TABLE V: AVERAGE OF TEN 10-FOLD CVS RESULTS (ERROR)

\begin{tabular}{llll}
\hline $\begin{array}{l}\text { Multiple } \\
\text { regression }\end{array}$ & Decision tree & $\begin{array}{l}\text { Overlapped } \\
\text { decision tree }\end{array}$ & $\begin{array}{l}\text { Proposed } \\
\text { method }\end{array}$ \\
\hline 0.1896 & 0.2319 & 0.2133 & 0.1728 \\
\hline
\end{tabular}

In Fig. 3, multiple regression analysis and our proposed method show comparatively few errors, but the decision tree and the overlapped decision tree have many errors. In most cases, the error of our proposed method is less than the error of multiple regression analysis.

In Fig. 4 the proposed method shows the fewest errors, and the multiple regression analysis shows the second fewest errors. The decision tree and the overlapped decision tree have more errors. Furthermore, as shown in Table V, the average error of the proposed method is 0.1728 , which is less than 0.05 compared to the decision tree. Our proposed method outperforms the other methods in terms of toxicity value prediction.

Fig. 5 and Fig. 6 and Table VI show the results of experiment 2, where the cosine similarity was evaluated instead of the average error in Figs. 3 and 4 and Table V.

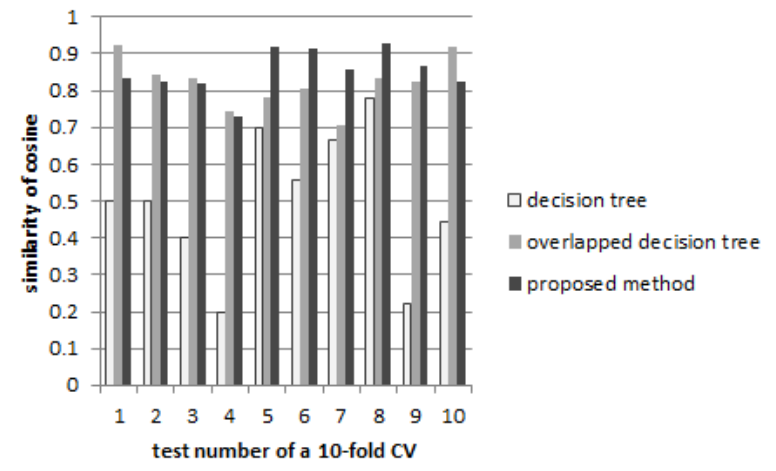

Fig. 5. 10-fold CV results (cosine).

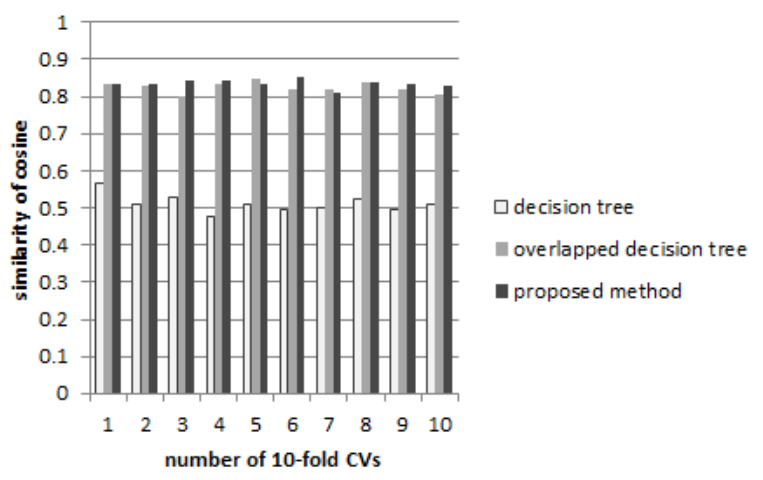

Fig. 6. Ten 10-fold CVs results (cosine).

TABLE VI: AVERAGE OF TEN 10-FOLD CVS RESUlTS (COSINE)

\begin{tabular}{lll}
\hline \hline Decision tree & $\begin{array}{l}\text { Overlapped } \\
\text { decision tree }\end{array}$ & $\begin{array}{l}\text { Proposed } \\
\text { method }\end{array}$ \\
\hline 0.5113 & 0.8253 & 0.8346 \\
\hline \hline
\end{tabular}

In Fig. 5, the overlapped decision tree and our proposed method have relatively stable similarity around $0.7-0.9$, and the decision tree has similarity over a wider range: $0.2-0.8$.

Fig. 6 shows that the overlapped decision tree and our proposed method have high similarity around $0.8-0.85$, in contrast, the decision tree has comparatively low similarity in 0.45-0.6. As shown in Table VI, the similarity of our proposed method is 0.8346 , which is higher than the similarity of the overlapped decision tree. Thus, higher similarity is shown when instances belong to more than one class rather than a single class.

In summary, our proposed method with defuzzification shows the highest accuracy when predicting the toxicity value in comparison with other typical methods. As for the toxicity classification problem, our proposed method can predict a class that closely resembles the actual class.

\section{CONCLUSION}

In this paper we discussed a framework for predicting 
toxicity by P450. Our proposed method is based on fuzzy decision trees since the amount of fluorescent metabolites is not allowed to have a crisp boundary point for classification. Our proposed method also considers the class ambiguity since the toxicity value to define each class is also not allowed to have crisp boundary points.

From the results of our experiments, our proposed method shows smaller average error than any other methods and higher cosine similarity. Therefore, our method, which considers the ambiguity, is superior to others for the prediction of toxicity.

\section{ACKNOWLEDGMENT}

This work was partly supported by Grant-in-Aid for Scientific Research (B) (24300056) from MEXT.

\section{REFERENCES}

[1] G. Chang, Y. Mori, S. Mori, T. Irie, H. Nagai, T. Goto, Y. Tatsu, K. Morigaki, and H. Imaishi, "Microarray of human P450 with an integrated oxygen sensing film for high-throughput detection of metabolic activities," Anal. Chem., vol. 84, 2012.

[2] K.-M. Lee et al., "A fuzzy decision tree induction method for fuzzy data," Fuzzy Systems Conference Proceedings, 1999.

[3] Y. Yuan and M. J. Shaw, "Induction of fuzzy decision tree," Fuzzy Sets and Systems, 1995 .

[4] H. Veith et al., "Comprehensive characterization of cytochrome P450 isozyme selectivity across chemical libraries," Nature biotechnology, 2009.

[5] G. Chang, K. Morigaki, Y. Tatsu, T. Hikawa, T. Goto, and H. Imaishi, "Vertically integrated human P450 and oxygen sensing film for the assays of P450 metabolic activities," Anal Chem., 2011.

[6] Y. Ueda, K. Morigaki, Y. Tatsu, N. Yumoto, and H. Imaishi, "Immobilization and activity assay of cytochrome P450 on patterned lipid membranes," Biochem Biophys Res Commun., 2007.

[7] K. Morigaki, K. Mizutani, E. Kanemura, Y. Tatsu, N. Yumoto, and H. Imaishi "Photoregulation of cytochrome P450 activity by using caged compound.," Anal Chem., 2012.

[8] L. A. Zadeh, "Fuzzy sets as a basis for a theory of possibility," Fuzzy sets and systems, 1999

[9] J. R. Quinlan, "C4.5: Programs for machine learning," Morgan Kaufmann, San-Mateo, California, 1993.

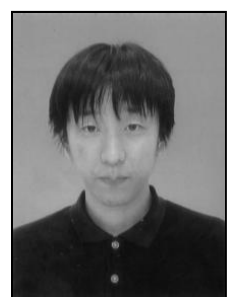

Daishi Yajima obtained his bachelor of engineering from Kobe University in 2013. He is currently a student of the master course at Graduate School of System Informatics, Kobe University (Kobe, Japan) His research interests include intelligent data processing and bioinformatics.

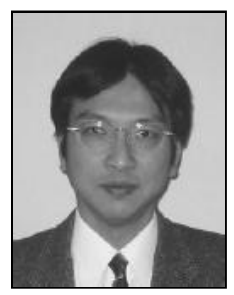

Takenao Ohkawa received his B.E, M.E., and Ph.D. degrees from Osaka University in 1986, 1988, and 1992 , respectively. He is currently a professor in the Department of Information Science, Graduate School of System Informatics, Kobe University. His research interests include intelligent data processing and bioinformatics. He is a member of the IEEE, the Information Processing Society of Japan, the Japanese Society for Bioinformatics, the Institute of Electronics, Information, and Communication Engineers, the Institute of Electrical Engineers in Japan, and the Japanese Society for Artificial Intelligence.

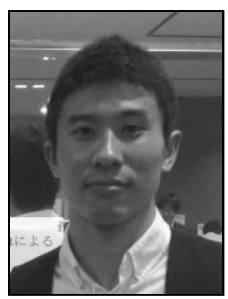

Kouhei Muroi obtained his bachelor of agriculture from Kobe University in 2013. He is currently a student of the master course at Graduate School of Agriculture, Kobe University (Kobe, Japan). His research interests include toxicology and environmental study.

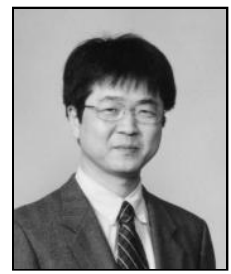

Hiromasa Imaishi received his Ph.D. degrees from Kyoto University in 1990 . He is currently a Professor in the Research Center for Environmental Genomics, Kobe University. His research interests include toxicology and environmental study. $\mathrm{He}$ is a member of the Japanese Biochemical Society, the Molecular Biological Society of Japan, the Japan Society for Bioscience Biotechnology and Agrochemistry, the chemical society of Japan and the Biological Society 